\title{
Aleitamento Materno Exclusivo e introdução de alimentos industrializados nos primeiros dois anos de vida
}

\author{
Exclusive Breastfeeding and introduction of processed foods \\ in the first two years of life
}

Lactancia Materna Exclusiva y la introducción de los alimentos procesados em los primeros dos años de vida

Rafael Alves Mata de Oliveira ${ }^{1}$ Fernanda Zanoni Cônsolo Karine de Cássia Freitas ${ }^{3}$ Giovana Eliza Pegolo ${ }^{4}$

${ }^{1}$ Nutricionista pela Universidade Federal de Mato Grosso do Sul (UFMS). Residente do Programa de Residência Multiprofissional em Cuidados Continuados Integrados: Hospital São Julião. E-mail: rafaelm004@gmail.com

${ }^{2}$ Doutora e Mestre pelo Programa de Pós-Graduação em Saúde e Desenvolvimento na Região Centro-Oeste, Universidade Federal de Mato Grosso do Sul (UFMS). Docente do Curso de Nutrição e do Programa de Residência Multiprofissional em Atenção ao Paciente Crítico (UFMS). E-mail: fernandazanoni@yahoo.com.br

${ }^{3}$ Doutora e Mestre em Ciências pela Universidade Federal de São Paulo (UNIFESP). Docente do Curso de Nutrição e do Programa de Pós-Graduação em Saúde e Desenvolvimento na Região Centro-Oeste, da Universidade Federal de Mato Grosso do Sul (UFMS). E-mail: kcfreitas@gmail.com

${ }^{4}$ Doutora em Alimentos e Nutrição pela Universidade Estadual Paulista (UNESP) e Mestre em Ciências pela Universidade de São Paulo (USP). Docente do Curso de Nutrição e do Programa de Residência Multiprofissional em Atenção ao Paciente Crítico, da Universidade Federal de Mato Grosso do Sul (UFMS).

E-mail: giovana.pegolo@hotmail.com 
Resumo: Objetivou-se identificar a frequência de Aleitamento Materno Exclusivo e a introdução de alimentos industrializados entre crianças com idade inferior a dois anos. Trata-se de estudo transversal, com amostra de conveniência, constituída por 31 mães. 61,3\% das mães informaram Aleitamento Materno Exclusivo, por período médio de 4,5 meses, contudo $86,9 \%$ e $67,8 \%$ das mães ofertaram água e chá, antes dos primeiros seis meses de vida, o que contraria a definição de aleitamento exclusivo. A oferta de alimentos industrializados antes dos dois anos foi mencionada por $96 \%$ das mães para sucos artificiais, e $87 \%$, para refrigerantes. Para macarrão instantâneo, doces, biscoitos recheados e salgadinhos, $88,2 \%, 89,3 \%, 86,4 \%$ e $80 \%$ registraram essa prática, respectivamente. Conclui-se que o Aleitamento Materno foi mencionado pela maioria das participantes, contudo não de forma exclusiva. A oferta de alimentos complementares mostrou-se inadequada, considerando a introdução de alimentos industrializados em idade precoce.

Palavras-chave: alimentação complementar; consumo de alimentos; leite materno.

Abstract: The objective was to identify the frequency of Exclusive Breastfeeding and the introduction of processed foods among children under two years of age. This is a cross-sectional study with a convenience sample composed of 31 mothers. It was found that $61.3 \%$ of the mothers reported Exclusive Breastfeeding for an average period of 4,5 months, however, $86.9 \%$ and $67.8 \%$ of the mothers offered water and tea, before first six months of life, which is contrary to exclusive breastfeeding' definition. The processed food's offer before two years was mentioned by $96 \%$ of mothers for artificial juices and $87 \%$ for soft drinks; this practice was recorded in $88.2 \%, 89.3 \%, 86.4 \%$ and $80 \%$, respectively, for instant noodles, sweets, cookies and chips. It's concluded that the Exclusive Breastfeeding was mentioned by the majority of the participants, but not exclusively. The supply of complementary foods proved to be inadequate, considering the introduction of industrialized foods at an early age.

Keywords: supplementary feeding; food consumption; milk human.

Resumen: Se objetivó identificar la frecuencia de la Lactancia Materna Exclusiva y la introducción de los alimentos procesados entre los niños menores de dos años. Se trata de um estudio transversal con una muestra de conveniencia compuesta por 31 madres. Se encontró que el $61,3 \%$ de madres informaron de la Lactancia Materna Exclusiva durante un período medio de 4,5 meses, sin embargo, 86,9\% y 67,8\% de las madres ofrecieron agua y té, antes de los primeros seis meses de vida, lo que contradice la definición de la Lactancia Materna Exclusiva. El suministro de alimentos procesados antes de dos años fue mencionada por el $96 \%$ de las madres para los jugos artificiales y el $87 \%$ para los refrescos; esta práctica se registro en el $88,2 \%, 89,3 \%, 86,4 \%$ y $80 \%$, respectivamente para los fideos instantáneos, los dulces, las galletas y los snacks. Llegamos a la conclusión de que la Lactancia Materna Exclusiva fue mencionado por la mayoría de las participantes, pero no de forma exclusiva. La oferta de alimentos complementarios resultó inadecuada, considerando la introducción de alimentos industrializados en edad precoz.

Palabras clave: alimentación suplementaria; consumo de alimentos; leche humana. 


\section{INTRODUÇÃO}

A Organização Mundial da Saúde (OMS) recomenda a adoção do Aleitamento Materno Exclusivo e em livre demanda nos primeiros seis meses de vida, preconização esta também adotada no Brasil. A definição de Aleitamento Materno Exclusivo abrange as seguintes especificações: leite materno, direto da mama ou ordenhado, ou leite humano de outras fontes, sem oferta de qualquer outro líquido ou sólido, com exceção de suplementos vitamínicos e/ou minerais ou medicamentos.

O aleitamento materno é uma prática desejada pelas mães por representar afeto e carinho, além de aumentar o vínculo entre a mãe e o bebê. Além disso, ultrapassa as vantagens associadas ao conteúdo nutricional, por acarretar uma série de benefícios para a saúde das crianças, especialmente pela redução do risco de infecções e por reduzir significativamente o risco de morbidade e mortalidade, principalmente por diarreia e pneumonia, nos primeiros anos de vida.

Estudos epidemiológicos e biológicos ratificam que a decisão de não amamentar repercute em efeitos importantes em longo prazo na saúde, nutrição e desenvolvimento da criança e na saúde da mãe. Além da redução do risco de morbidade e mortalidade, sabe-se que as crianças que são amamentadas por maior tempo apresentam maior inteligência do que aquelas amamentadas por períodos mais curtos ou não amamentadas. Tais desigualdades impactam ao longo da vida, inclusive com evidências de que a amamentação pode proteger contra o excesso de peso e diabetes na vida adulta.

As dificuldades observadas desde o início da amamentação, antes mesmo da alta hospitalar, podem resultar em menor duração do Aleitamento Materno Exclusivo, representando um dos fatores relacionados ao desmame precoce. Nesse contexto, a introdução de outros tipos de leite que não o materno e a oferta de alimentos nos primeiros seis meses de vida contribuem para o rompimento do Aleitamento Materno Exclusivo. Outra questão que deve ser alvo de atenção corresponde ao fato de que o primeiro ano de vida é considerado um período crítico para desenvolvimento da obesidade, com tendência à manutenção na vida adulta, paralelo a comorbidades como o diabetes e doenças cardiovasculares. Assim, a introdução precoce 
de alimentos, como os industrializados, recentemente também classificados como processados e ultraprocessados, potencializa o risco para o desenvolvimento de obesidade. Tem-se ainda a preocupação com a introdução de alimentos com conteúdo nutricional insatisfatório justamente em fase tão importante para a formação do hábito alimentar.

Tendo em vista a importância do Aleitamento Materno Exclusivo, nosso objetivo foi identificar a frequência de Aleitamento Materno Exclusivo e a idade de introdução de alimentos industrializados nos primeiros dois anos de vida.

\section{MATERIAL E MÉTODOS}

Trata-se de um estudo transversal constituído por amostra de conveniência, com abordagem quantitativa e qualitativa, realizado em um Centro de Educação Infantil de Campo Grande (Mato Grosso do Sul).

Integraram a amostra mães de crianças com idade até 2 anos, 11 meses e 29 dias na data de coleta dos dados e que apresentaram idade superior a 18 anos. Os critérios de exclusão foram: mães que por algum motivo não estavam presentes nos dias de coleta de dados, mães com menos de 18 anos de idade e cujos filhos apresentassem idade superior à faixa de idade pré-determinada.

Para caracterização das participantes, foram consideradas as seguintes informações sociodemográficas e relacionadas à gestação: idade; escolaridade materna; estado civil e retorno ao trabalho no primeiro ano de vida da criança; licença maternidade; período de licença; número de gestações; número de consultas pré-natal; amamentação na primeira hora de vida e orientação pós-parto sobre aleitamento.

Especificamente sobre a oferta de leite materno, foram investigadas as seguintes informações: oferta de leite materno; oferta de leite em pó industrializado e/ou fórmula infantil; duração do Aleitamento Materno Exclusivo; doação do leite materno e a percepção da nutriz quanto à importância do leite materno para a criança e para a mãe.

Sobre a introdução de líquidos e alimentos, foram considerados os seguintes itens: água, chá, açúcar, refrigerante, sucos artificiais, macarrão instantâneo, doces, biscoitos recheados e salgadinhos industrializados; e 
respectiva idade de introdução, considerando os primeiros dois anos de vida. Realizou-se pré-teste do instrumento de pesquisa, com mães de crianças na mesma faixa de idade e não pertencentes ao local de pesquisa, a fim de confirmar o entendimento das questões pelo público-alvo.

Para a identificação da Classificação Econômica, foi adotado o Critério de Classificação Econômica da Associação Brasileira de Empresas de Pesquisas (ABEP, 2015). Os pontos de corte, em ordem decrescente de nível econômico (associados às estimativas de renda domiciliar mensal), foram = A: 45 a 100 pontos (R\$20.272,56); B1: 38 a 44 pontos (R\$ 8.695,88); B2: 29 a 37 pontos (R\$ 4.427,00); C1: 23 a 28 pontos (R\$ 2.409,01); C2: 17 a 22 pontos (R\$1.446,24); e D-E: 0 a 16 pontos (R\$ 639,78).

Quanto à análise estatística, os dados obtidos foram analisados de forma descritiva e quantitativa, sendo a última análise realizada pelo teste qui-quadrado (com correção de Yates), calculado com o emprego do programa BioEstat 5.3, fixando-se em 5\% o nível de rejeição da hipótese de nulidade. As questões qualitativas foram transcritas a fim de expressar o conhecimento/sentimento associado.

O presente estudo foi aprovado pelo Comitê de Ética em Pesquisas com Seres Humanos (Parecer número 1.328.134) da Universidade Federal de Mato Grosso do Sul (UFMS). As mães manifestaram concordância de sua participação voluntária por meio da assinatura do Termo de Consentimento Livre e Esclarecido (TCLE).

\section{RESULTADOS E DISCUSSÃO}

A amostra foi constituída por 31 mães, representando 73,8\% do total ( $n=42$ ) elegível (2,4\% das mães não aceitaram participar e $23,8 \%$ não compareceram na unidade de ensino no período de coleta de dados ou não devolveram o TCLE).

A idade média das participantes foi de 31,6 anos $( \pm 6,6)$. No que diz respeito à escolaridade, 35,5\% relataram possuir Ensino Médio Completo. Para os demais níveis de escolaridade, foram observadas as seguintes proporções: Ensino Superior Completo, 19,3\%; Ensino Superior Incompleto, 19,3\%; Ensino Fundamental Incompleto, 9,7\%; Pós-Graduação, 9,7\%; Ensino Fundamental Completo, 3,2\%, e Ensino Médio Incompleto, 3,2\%. A maioria 
das participantes (64,5\%), no primeiro ano de vida do filho, relatou estado civil casado. Entre as demais, 25,8\% informaram estado civil solteiro, e 9,7\%, divorciado/separado.

Para a classificação econômica, foi constatada a seguinte distribuição: $38,7 \%$ das participantes foram identificadas na Classe B2; $25,8 \%$ e 22,6\% nas Classes C1 e C2, respectivamente; e 6,5\% na Classe B1. Nas Classes A e D-E foram observados percentuais de 3,2\% em cada estrato.

A Tabela 1 apresenta as características relacionadas ao período gestacional e ao aleitamento materno. Destaca-se a proporção de mães (61,3\%) que relataram Aleitamento Materno Exclusivo. Nesse sentido, embora realizada em 2009, a II Pesquisa de Prevalência de Aleitamento Materno apresentou, para aquele momento, o cenário de adoção ao aleitamento para todas as capitais brasileiras. Campo Grande apresentou prevalência de Aleitamento Materno Exclusivo de 50,1\%. Outras capitais, como São Paulo e Rio de Janeiro, alcançaram 39,1\% e 40,7\%, respectivamente, e a média nacional foi de $41 \%$ (BRASIL, 2009), valores inferiores ao identificado na presente amostra.

Segundo relatório, com abrangência mundial, publicado em 2017, pelo Fundo das Nações Unidas para a Infância (UNICEF) e pela OMS, apenas 40\% das crianças com menos de seis meses de idade recebem exclusivamente leite materno. Além disso, destaca-se que apenas 23 dos 194 países analisados apresentaram índices de Aleitamento Materno Exclusivo até os seis meses acima de 60\%. No Brasil, o índice de amamentação exclusiva entre as crianças com até seis meses é de 38,6\% (ORGANIZAÇÃO DAS NAÇÕES UNIDAS [ONU], 2017). 
Tabela 1 - Características das mães de uma amostra de crianças matriculadas em um Centro de Educação Infantil sobre período gestacional e aleitamento materno. Campo Grande, MS, 2016

\begin{tabular}{l|c|c}
\hline Características relacionadas ao período gestacional e & Sim & Não \\
\cline { 2 - 3 } ao Aleitamento Materno & $\mathbf{n ~ ( \% )}$ & $\mathbf{n ~ ( \% )}$ \\
\hline Trabalhou fora no 10 ano de vida & $20(64,5)$ & $11(35,5)$ \\
Licença Maternidade & $19(61,3)$ & $12(38,7)$ \\
Primeira gestação & $8(25,8)$ & $23(74,2)$ \\
Realizou pré-natal & $31(100,0)$ & $0(0,0)$ \\
Amamentou na primeira hora após o parto & $25(80,6)$ & $6(19,4)$ \\
Orientação da maternidade após o parto sobre aleitamento & $29(93,5)$ & $2(6,5)$ \\
Doação do leite materno & $4(12,9)$ & $27(87,1)$ \\
Sabe a importância do leite materno para a criança & $29(93,5)$ & $2(6,5)$ \\
Sabe a importância do leite materno para a mãe & $16(51,6)$ & $15(48,4)$ \\
Realizou Aleitamento Materno Exclusivo & $19(61,3)$ & $12(38,7)$ \\
\hline
\end{tabular}

Informações complementares apontam que o Aleitamento Materno Exclusivo apresentou duração média de 4,5 meses $( \pm 2,0)$. Além disso, observou-se licença maternidade de 4,7 meses $( \pm 0,9)$, número médio de filho de 2,6 $( \pm 0,9)$ e, em média, 10,9 consultas de pré-natal $( \pm 4,0)$.

Nota-se que a duração em meses do Aleitamento Materno Exclusivo mostrou-se inferior ao período proposto pela OMS, porém, próximo ao período médio informado de licença maternidade, o que pode sugerir que pouco antes de retornar ao trabalho as mães já iniciaram o desmame.

Sobre o período de Aleitamento Materno Exclusivo, outros levantamentos também apontam períodos inferiores ao preconizado e ainda mais desfavoráveis. No Estado de São Paulo, em um Hospital Universitário, foi identificada duração média de Aleitamento Materno Exclusivo de 4 meses $( \pm 2,3)$ (CAMPOS et al., 2015). Dados obtidos durante uma campanha de vacinação de crianças menores de um ano, em 2011, em São Paulo, revelaram período de 2,65 meses de Aleitamento Exclusivo (TAMASIA; VENÂNCIO; SALDIVA, 2015). Pesquisa com crianças de quatro a nove meses, em capitais de três regiões brasileiras, identificou que a duração média do Aleitamento Materno Exclusivo foi de 3,4 meses $( \pm 1,7)$ (SOUZA et al., 2014). Por outro lado, entre bebês chilenos, a duração média foi de 8,6 meses $( \pm 3,7)$ (PINO et al., 2013). 
No que diz respeito à oferta de outros tipos de leite que não o leite materno, observou-se que $38,7 \%$ das mães $(n=12)$ ofereceram leite em pó industrializado, fórmula infantil ou de origem animal in natura nos primeiros 6 meses.

Ao avaliar a alimentação complementar de lactentes de 4 a 9 meses de vida, de três capitais brasileiras (São Paulo, Curitiba, Recife), constatou-se que, entre as crianças que recebiam outros tipos de leite, 52,5\% recebiam leite de vaca integral, e $41 \%$ recebiam fórmulas infantis ou de seguimento para lactentes (SOUZA et al., 2014).

Entre lactantes italianos, aos seis meses de vida, 73\% estavam recebendo leite materno, contudo apenas $7 \%$ de forma exclusiva. Entre as demais crianças, $40 \%$ estavam recebendo fórmulas infantis e leite materno, sendo que $27 \%$ estavam recebendo apenas fórmulas infantis, e $26 \%$, leite materno e alimentos complementares (CARLETTI et al., 2017).

Sobre a importância do aleitamento imediato após o parto, é amplamente reconhecido o benefício do colostro em função da presença de inúmeras substâncias imunológicas protetoras contra infecções - como infecções respiratórias, diarreia e septicemia neonatal (PALMEIRA; CARNEIROSAMPAIO, 2016). Na amostra estudada, $80,6 \%$ das mães amamentaram na primeira hora após o parto.

Em 2009, de acordo com a II Pesquisa de Prevalência de Aleitamento Materno, 74,3\% das crianças menores de um ano em Campo Grande foram amamentadas na primeira hora de vida. Para as capitais da Região CentroOeste, as prevalências observadas foram: Cuiabá, 77,4\%; Distrito Federal, 72,5\%, e Goiânia, 66,7\%. A prevalência nacional de aleitamento materno na primeira hora de vida foi de 67,7\% (BRASIL, 2009).

Em Registro, município do interior de São Paulo, 81,1\% das mães relataram a amamentação na primeira hora de vida do bebê (TAMASIA; VENÂNCIO; SALDIVA, 2015). Sá et al. (2016), no Distrito Federal, constataram que $77,3 \%$ das mães mencionaram amamentação na primeira hora de vida. Porém os autores ressaltam que, entre as mães que não amamentaram, os fatores que interferiram negativamente no aleitamento materno na primeira hora de vida referiram-se à prestação da assistência ao pré-natal, especialmente à não realização do pré-natal de forma adequada, ao parto cesário e 
ao fato de mãe e filho não permanecerem em alojamento conjunto após o parto. As proporções citadas acima aproximam-se da proporção verificada neste trabalho.

A Tabela 2 retrata a idade de introdução de alimentos/produtos industrializados nos dois primeiros anos de vida.

Inicialmente é importante destacar a observação de resultados contraditórios a respeito da oferta de líquidos nos primeiros seis meses, pois $86,9 \%$ das mães mencionaram oferta de água, e 67,8\% de chá, proporções superiores a de mães que mencionaram Aleitamento Materno Exclusivo (61,3\%), o que nos conduz a sugerir rompimento com os critérios que definem o Aleitamento Materno Exclusivo, ou seja, possível desconhecimento do que constitui a recomendação.

Tabela 2 - Idade de introdução de líquidos e alimentos/produtos industrializados nos dois primeiros anos de vida de crianças $(n=31)$ matriculadas em um Centro de Educação Infantil. Campo Grande, MS, 2016

\begin{tabular}{l|c|c|c|c|c}
\hline \multirow{2}{*}{ Alimentos/produtos } & \multicolumn{5}{c}{ Idade de introdução } \\
& \multicolumn{5}{c|}{$\mathbf{n}$ (\%) } \\
\cline { 2 - 7 } & $\begin{array}{c}\text { Primeiros } \\
\mathbf{6} \text { meses }\end{array}$ & $\begin{array}{c}\text { De 7 a 11 } \\
\text { meses }\end{array}$ & $\begin{array}{c}\text { De 12 a } \\
\text { 23 meses }\end{array}$ & $\begin{array}{c}\text { A partir } \\
\text { de 24 } \\
\text { meses }\end{array}$ & $\begin{array}{c}\text { Não } \\
\text { lembra }\end{array}$ \\
\hline Água (n=31) & $27(86,9)$ & $4(12,9)$ & $0(0,0)$ & $0(0,0)$ & $0(0,0)$ \\
Chá $(n=28)$ & $19(67,8)$ & $8(28,6)$ & $1(3,6)$ & $0(0,0)$ & $0(0,0)$ \\
Açúcar (n=26) & $7(26,9)$ & $8(30,8)$ & $11(42,3)$ & $0(0,0)$ & $0(0,0)$ \\
Refrigerantes (n=23) & $0(0,0)$ & $1(4,4)$ & $19(82,6)$ & $3(13,0)$ & $0(0,0)$ \\
Suco Artificial (n=25) & $2(8,0)$ & $6(24,0)$ & $16(64,0)$ & $1(4,0)$ & $0(0,0)$ \\
Macarrão Instantâneo (n=17) & $0(0,0)$ & $2(11,8)$ & $13(76,4)$ & $2(11,8)$ & $0(0,0)$ \\
Doces (n=28) & $0(0,0)$ & $5(17,9)$ & $20(71,4)$ & $3(10,7)$ & $0(0,0)$ \\
Biscoitos Recheados (n=22) & $0(0,0)$ & $2(9,1)$ & $17(77,3)$ & $3(13,6)$ & $0(0,0)$ \\
Salgadinhos Industrializados (n=20) & $0(0,0)$ & $2(10,0)$ & $14(70,0)$ & $4(20,0)$ & $0(0,0)$ \\
\hline
\end{tabular}

Nota: Número variável para cada líquido ou alimento/produto de acordo com as respostas afirmativas.

No que diz respeito ao conhecimento sobre o que compreende o Aleitamento Materno Exclusivo, foram constatadas, com mães atendidas em uma instituição que zela pela promoção ao aleitamento exclusivo, práticas 
equivocadas especialmente em relação à introdução precoce e inadequada de leite e/ou líquidos antes dos seis meses (CAMPOS et al., 2015).

Outra constatação relevante sobre a introdução de alimentos refere-se ao açúcar. A oferta foi relatada por $26,7 \%$ das mães nos primeiros seis meses de vida (Tabela 2). Além disso, 83,8\% ofertaram açúcar antes dos primeiros dois anos de vida, prática compreendida como de risco nutricional, tendo em vista tratar-se de ingestão apenas de energia, isenta de nutrientes, e possivelmente em quantidade excessiva. Recomenda-se que a ingestão não ultrapasse $10 \%$ da ingestão diária de energia, em todas as fases da vida (OMS, 2015).

Enquanto o Aleitamento Materno Exclusivo é um fator protetor da obesidade, a introdução precoce de açúcar e alimentos açucarados é um fator de risco para o ganho de peso em crianças (SIMON; SOUZA; SOUZA, 2009). Nesse sentido, atualmente o consumo de alimentos industrializados, muitas vezes com quantidades expressivas de açúcar, está cada vez mais precoce, inclusive por crianças com menos de dois anos. Entre crianças de seis meses a um ano de idade, 75,6\% receberam no mínimo um alimento não saudável no dia anterior à entrevista, sendo que 40,4\% das mães mencionaram a oferta de alimentos açucarados, 63,7\%, de biscoitos, e, 7,1\%, de refrigerantes (TAMASIA; VENÂNCIO; SALDIVA, 2015).

Nossos resultados revelam que parcelas expressivas de crianças consumiram refrigerantes (87\%) e sucos artificiais (96\%) antes dos dois anos de idade. A oferta de doces, macarrão instantâneo e biscoitos recheados também foi observada, com proporções, respectivamente, de 89,3\%, 88,2\% e $86,4 \%$ de crianças com consumo nessa faixa de idade (Tabela 2).

Uma análise do consumo de alimentos de 218 crianças, assistidas na Atenção Básica de Macaé, RJ, revelou que a oferta de açúcar esteve presente em 23,8\% das crianças de 6 a 12 meses de idade. Os refrigerantes já tinham sido oferecidos para $17,8 \%$ das crianças nessa mesma faixa de idade (MARINHO et al., 2016).

Uma das recomendações do Guia Alimentar para crianças menores de dois anos é evitar o consumo de alimentos industrializados, por serem constituídos, na maioria das vezes, por quantidades excessivas de açúcar, sal e gorduras. O consumo desses alimentos e líquidos, como refrigerantes, 
salgadinhos, doces e sucos artificiais, está associado diretamente ao excesso de peso, anemia e alergias alimentares (BRASIL, 2013).

Especificamente em relação ao açúcar, ao analisar a idade de introdução entre as 26 mães que afirmaram esta prática, não foi detectada diferença significativa $(p=0,3960)$ em relação à classificação econômica (Tabela 3). Contudo nota-se que as mães que ofereceram o açúcar antes dos seis meses foram as pertencentes às classes B1, B2 e C1, com estimativas de renda mensal entre $R \$ 2.409,01$ a $R \$ 8.695,88$, valores que superam substancialmente o salário mínimo vigente.

Tabela 3 - Idade de introdução de açúcar e a classificação econômica materna. Campo Grande, MS, 2016

\begin{tabular}{|c|c|c|c|c|c|c|}
\hline \multirow{2}{*}{$\begin{array}{c}\text { Idade de introdução } \\
\text { de açúcar }\end{array}$} & \multicolumn{6}{|c|}{ Classificação econômica } \\
\hline & A & B1 & B2 & C1 & C2 & D-E \\
\hline Antes dos 6 meses & $0(0,0)$ & $1(3,8)$ & $1(3,8)$ & $1(3,8)$ & $0(0,0)$ & $0(0,0)$ \\
\hline Do 6 ao 11 mês & $1(3,8)$ & $1(3,8)$ & $6(23,1)$ & $1(3,8)$ & $2(7,7)$ & $1(3,8)$ \\
\hline A partir de 12 meses & $0(0,0)$ & $0(0,0)$ & $3(11,5)$ & $5(19,2)$ & $3(11,5)$ & $0(0,0)$ \\
\hline
\end{tabular}

Entre os alimentos industrializados, chama a atenção a oferta precoce de sucos, tanto na forma em pó quanto dos sucos em caixa. Para a idade de introdução, nota-se a oferta antes dos seis meses por $8 \%$ das mães, sendo que $88 \%$ ofertaram antes dos dois anos de vida da criança (Tabela 2). Quanto à classificação econômica, não foi constatada diferença significativa entre a idade de introdução de suco artificial $(p=0,7428)$ e classificação econômica.

Sobre o consumo de bebidas açucaradas, análises derivadas da Pesquisa Nacional de Saúde (PNS), de 2013, constataram alta prevalência de consumo de bebidas açucaradas por crianças brasileiras menores de dois anos, sendo que essa prática mostrou-se associada às características sociodemográficas e hábitos familiares. Para refrigerantes, o consumo no dia anterior foi referido por cerca de uma em cada três crianças (JAIME; PRADO; MALTA, 2017).

Uma pesquisa com crianças de creches públicas de São Paulo demonstrou que mães com renda familiar inferior a um salário mínimo apresentaram duas vezes maior risco de introduzirem precocemente refri- 
gerantes e salgadinhos na dieta de seus filhos. Os autores concluíram que são os filhos de mães com mais baixa renda que estão mais propensos a consumirem alimentos industrializados nos primeiros anos de vida (TOLONI et al., 2011). Esse perfil de consumo também foi observado entre crianças do interior de Minas Gerais. A introdução de alimentos industrializados/ supérfluos mostrou-se expressiva, pois, apesar de renda per capita mensal de $R \$ 140,00,80,2 \%$ das mães e/ou responsáveis afirmaram ter oferecido um ou mais alimentos aos filhos ainda com idade inferior a um ano (HEITOR; RODRIGUES; SANTIAGO, 2011).

Complementando a investigação dos fatores associados à amamentação, entre as mães que mencionaram Aleitamento Materno Exclusivo ( $n=19)$, não houve diferença significativa quanto ao período de aleitamento e a classe econômica ( $p=0,4422)$ e entre o Aleitamento Materno Exclusivo e as idades maternas $(p=0,4114)$ (Tabelas 4 e 5).

De qualquer forma, é possível notar que as mães identificadas nas classes com estimativa de renda menor (C2 e D-E) mantiveram o Aleitamento Materno Exclusivo por período inferior a seis meses com maior frequência do que as mães que informaram período igual ou superior a seis meses. 0 mesmo também foi observado para as mães da classe B2 (Tabela 4).

Tabela 4 - Período de Aleitamento Materno Exclusivo e classificação econômica materna. Campo Grande, MS, 2016

\begin{tabular}{l|c|c|c|c|c|c}
\hline \multirow{2}{*}{ Aleitamento Materno } & \multicolumn{6}{|c}{ Classificação econômica } \\
\cline { 2 - 7 } & A & B1 & B2 & C1 & C2 & D-E \\
\hline Por período igual ou & $0(0,0)$ & $2(10,5)$ & $3(15,8)$ & $2(10,5)$ & $1(5,3)$ & $0(0,0)$ \\
maior a 6 meses & $1(5,3)$ & $0(0,0)$ & $4(21,0)$ & $2(10,5)$ & $3(15,8)$ & $1(5,3)$ \\
Por período <6 meses
\end{tabular}

Qui-quadrado $(p=0,442)$.

Sobre a influência da renda no período de aleitamento, considerando o número de meses, 54,7\% das mães de uma maternidade de Joinville, SC, que ofereceram leite materno exclusivamente aos seus bebês tinham renda familiar menor de três salários mínimos, sendo R\$ 678,00/salário à época da obtenção dos dados (CONTARATO et al., 2016). 
Cabe registrar que a amamentação é considerada um dos poucos comportamentos de saúde positivos mais frequentes nos países pobres do que nos ricos. As mulheres com menor renda amamentam por mais tempo nos países de renda baixa e média. Tais resultados sugerem que os padrões de amamentação estão contribuindo para a redução das disparidades em saúde entre as crianças, as quais seriam maiores na ausência da amamentação (VICTORA et al., 2016).

Mesmo não tendo sido detectada diferença significativa entre período de Aleitamento Materno Exclusivo e a idade materna, nota-se que mães com idades entre 30 a 35 anos e superior a 35 anos informaram período inferior a seis meses, com maior frequência que aquelas que informaram período igual ou maior a seis (Tabela 5). Pode-se sugerir que a maior idade não representou maior adoção ao Aleitamento Materno Exclusivo, mesmo tendo-se como expectativa a possibilidade de não ser o primeiro filho e apresentar conhecimento prévio sobre as repercussões positivas do leite materno nos primeiros seis meses.

Tabela 5 - Período de Aleitamento Materno Exclusivo e idade materna. Campo Grande, MS, 2016

\begin{tabular}{l|c|c|c|c}
\hline \multirow{2}{*}{ Aleitamento Materno Exclusivo } & \multicolumn{4}{|c}{ Idade materna } \\
\cline { 2 - 5 } & $\begin{array}{c}\mathbf{2 0} \mathbf{a}< \\
\mathbf{2 5} \text { anos }\end{array}$ & $\begin{array}{c}\mathbf{2 5} \mathbf{a}< \\
\mathbf{3 0} \text { anos }\end{array}$ & $\begin{array}{c}\mathbf{3 0} \mathbf{\mathbf { a }}< \\
\mathbf{3 5} \text { anos }\end{array}$ & $\begin{array}{c}\mathbf{3} \mathbf{3 5} \\
\text { anos }\end{array}$ \\
\hline Por período igual ou maior a 6 meses & $1(5,3)$ & $3(15,8)$ & $1(5,3)$ & $3(15,8)$ \\
Por período $<6$ meses & $2(10,5)$ & $1(5,3)$ & $4(21,0)$ & $4(21,0)$ \\
\hline
\end{tabular}

Qui-quadrado $(p=0,4114)$.

Não foi detectada diferença significativa entre período de Aleitamento Materno Exclusivo (inferior a seis meses ou igual/superior a seis meses) e escolaridade da mãe $(p=0,8685)$. Outras pesquisas também investigaram essa associação. Em um Hospital-Escola do Rio Grande do Sul identificou-se que quanto mais anos de escolaridade da mãe, maior era o tempo pretendido para a amamentação exclusiva. Esses achados indicam que o acesso e conhecimento sobre a importância do aleitamento materno ainda estão relacionados ao nível de escolaridade, já que hábitos como a oferta de água 
e chá antes dos seis meses e inverdades, como a existência de leite fraco, são práticas e opiniões muito difundidas na população em geral (MACHADO et al., 2014).

Em uma maternidade de Joinville, SC, a maioria das mães que amamentaram exclusivamente seus filhos tinham mais de nove anos de escolaridade (67\%), ou seja, tinham no mínimo o ensino fundamental completo, mas não houve associação estatística entre a escolaridade e o Aleitamento Materno Exclusivo (CONTARATO et al., 2016). Embora no presente estudo não tenha sido constatada diferença significativa entre escolaridade e Aleitamento Materno Exclusivo, constatou-se que 54,8\% das mães apresentavam mais de 9 anos de escolaridade (35,5\% com Ensino Médio Completo e 19,3\% com Ensino Superior Completo).

Dentre as mães que relataram ( $n=14)$ os motivos para não amamentar ou interromper o Aleitamento Materno Exclusivo antes dos primeiros seis meses, as principais respostas foram: "retorno ao trabalho" ( $n=4)$, "não tinha leite suficiente" ( $n=2)$, "oferta de outro leite desde recém-nascido" $(n=1)$, "pouco leite" $(n=1)$, "faltou leite a partir do primeiro mês" $(n=1)$, "o leite secou" ( $n=1)$, "só amamentei por uma semana, pois o leite secou (associado ao uso de medicação)" ( $n=1$ ), "não desceu leite (justificado por patologias)" ( $n=1)$, "não tive leite (apenas por um mês)" ( $n=1)$ e "prematuro (internação)" (n=1).

Em uma pesquisa no interior do Estado de São Paulo, foram entrevistadas 12 mães atendidas em Unidades de Saúde da Família, e a experiência do desmame precoce não foi algo planejado e desejado, o que se confirma quando relataram o sentimento de tristeza em relação ao final do aleitamento materno. Foram registrados sentimentos de derrota e incapacidade ao não amamentar, o que gera um sentimento de culpa. Além disso, foram identificadas, por meio dos relatos das mães, algumas inverdades em relação ao leite materno, como: leite materno é fraco, não sustenta, causa cólicas no bebê (PRADO; FABBRO; FERREIRA, 2016).

A respeito da importância do leite materno para a criança, dentre as 29 mães que registraram resposta, os principais relatos foram: "o leite é o alimento mais completo"; "estreita os laços entre a mãe e o bebê"; "ajuda no desenvolvimento"; "auxilia na imunidade"; "ajuda o sistema imunológico 
Aleitamento Materno Exclusivo e introdução de alimentos industrializados nos primeiros dois anos de vida

e respiratório"; "fortalece e dá saúde à criança prevenindo de vários tipos de doenças"; "o leite materno possui todos os nutrientes"; "tem fácil digestão e protege o bebê de bactérias"; "ajuda no ganho de peso, protegendo de doenças, desenvolvimento da face, dos dentes, da fala e boa respiração" e "além do carinho e proteção, amamentar é vida, no leite materno se encontra tudo o que a criança necessita". As respostas nos conduz a considerar que as mães tinham conhecimento sobre os benefícios do aleitamento materno para seus filhos.

Das 16 mães que responderam a importância do aleitamento materno para a mãe, os principais motivos alegados foram: "traz satisfação e carinho"; "ajuda na recuperação pós-parto"; "traz contato e afeto"; "ajuda no relacionamento entre mãe e filho"; "ajuda a emagrecer, reestrutura o útero, organismo e afeto"; "diminui o risco de câncer de mama e diminui o risco de engravidar"; "a amamentação é mais econômica para a família"; e "diminui a incidência de depressão pós-parto". Nota-se que as respostas mostraram-se concordantes com os impactos positivos do aleitamento para a saúde da mãe.

Prado, Fabbro e Ferreira (2016) observaram que a importância e o efeito protetor do leite materno para a criança foram percebidos pela maioria das mães entrevistadas. Alguns dos benefícios relatados pelas mães para seus filhos foram: prevenção de doenças, desenvolvimento da dentição e redução dos custos da família com a compra de alimentos ou outros tipos de leites. Além disso, notou-se que a prática do Aleitamento Materno Exclusivo destacou-se por oferecer vantagens para as crianças e para a lactante, principalmente no pós-parto imediato.

Em uma pesquisa exploratória, com enfoque qualitativo, conduzida com dez puérperas, observou-se que, quando questionadas sobre a importância do aleitamento materno para os bebês, os principais relatos foram: previne doenças; promove o desenvolvimento da criança; tem todos os nutrientes que o bebê necessita. Tais falas ressaltam os aspectos biológicos associados à amamentação e revelam um comprometimento velado de responsabilização da mulher pela saúde do filho. Quando questionadas quanto aos benefícios da amamentação para as mães, disseram que o aleitamento diminui o risco de câncer de mama e de útero. Os resultados, de acordo com 
os autores, demonstraram que as mulheres sentem-se fortemente influenciadas por aspectos culturais, como a relevância da opinião de familiares e profissionais da saúde, por experiências anteriores em aleitar, pela influência dos padrões estéticos de beleza e ainda pela construção de laços afetivos por meio do aleitamento materno, o que representa culturalmente a figura da boa mãe como aquela que amamenta (JUNGES et al., 2010).

\section{CONCLUSÃO}

A maioria (61,3\%) das mães informaram Aleitamento Materno Exclusivo, por período médio de 4,5 meses, contudo parcelas substanciais informaram consumo de água e chá antes dos primeiros seis de meses vida, o que contraria a definição de Aleitamento Materno Exclusivo. Embora em proporção menor de mães, a oferta de açúcar também foi registrada antes dos seis meses. Além disso, a oferta de leite em pó industrializado ou de origem animal in natura nos primeiros 6 meses foi citada por mais de um terço das mães.

No que diz respeito à introdução de alimentos industrializados, a maioria das mães mencionou tal prática antes dos dois primeiros anos de vida, o que representa preocupação, pois a primeira fase da infância, após o período de aleitamento materno, é um momento importante para a formação de hábitos alimentares que poderão se perpetuar ao longo da vida.

Diante do exposto, estratégias de promoção do Aleitamento Materno Exclusivo, acompanhada de esclarecimentos sobre sua definição e benefícios, constituem ações essenciais para a saúde das mães e das crianças. Destaca-se também a importância da introdução da alimentação complementar de forma adequada, visando especialmente evitar o desenvolvimento de carências nutricionais e de doenças associadas à alimentação.

\section{REFERÊNCIAS}

ASSOCIAÇÃO BRASILEIRA DE EMPRESAS DE PESQUISA (ABEP). Critério de Classificação Econômica Brasil. 2015. Disponível em: <http://www.abep.org/ criterio-brasil>. Acesso em: 24 dez. 2015.

BRASIL. Ministério da Saúde. Dez passos para uma alimentação saudável: guia alimentar para crianças menores de dois anos: um guia para o profissional da saúde na atenção básica. 2. ed. Brasília: Ministério da Saúde, 2013. Disponível em: <http:// 
Aleitamento Materno Exclusivo e introdução de alimentos industrializados nos primeiros dois anos de vida

www.blog.saude.gov.br/images/arquivos/dez_passos_alimentacao_saudavel_guia. pdf>. Acesso em: 22 ago. 2017.

BRASIL. Ministério da Saúde. II Pesquisa de prevalência de aleitamento materno nas capitais brasileiras e Distrito Federal. Brasília: Ministério da Saúde, 2009. Disponível em: <http://bvsms.saude.gov.br/bvs/publicacoes/pesquisa_prevalencia_ aleitamento_materno.pdf>. Acesso em: 23 ago. 2017.

CAMPOS, A. M.; CARMONA, E. V.; HIGA, R.; VALE, I. N. Prática de aleitamento materno exclusivo informado pela mãe e oferta de líquidos aos seus filhos. Revista Latino-Americana de Enfermagem, Ribeirão Preto, SP, v. 23, n. 2, p. 283-90, mar./ abr. 2015.

CARLETTI, C.; PANI, P.; MONASTA, L.; KNOWLES, A.; CATTANEO, A. Introduction of complementary foods in a cohort of infants in northeast Italy: Do Parents comply with WHO Recommendations? Nutrients, Switzerland, v. 9, n. 1, p. 34-45, jan. 2017.

CONTARATO, A. A. P. F.; ROCHA, E. D. M.; CZARNOBAY, S. A.; MASTROENI, S. S. B.; VEUGELERS, P. J.; MASTROENI, M. F. Efeito independente do tipo de aleitamento no risco de excesso de peso e obesidade em crianças entre 12-24 meses de idade. Cadernos de Saúde Pública, Rio de Janeiro, v. 32, n. 12, p. 45-56, dez. 2016.

HEITOR, S. F. D.; RODRIGUES, L. R.; SANTIAGO, L. B. Introdução de alimentos supérfluos no primeiro ano de vida e as repercussões nutricionais. Ciência, Cuidado em Saúde, Maringá, PR, v. 10, n. 3, p. 430-6, jul./set. 2011.

JAIME, P. C.; PRADO, R. R. do; MALTA, D. C. Influência familiar no consumo de bebidas açucaradas em crianças menores de dois anos. Revista de Saúde Pública, São Paulo, v. 51, Supl. 1, p. 1-13, jun. 2017.

JUNGES, C. F.; RESSEL, L. B.; BUDÓ, M. L. D.; PADOIN, S. M. de; HOFFMANN, I. C.; SEHNEM, G. D. Percepções de puérperas quanto aos fatores que influenciam o aleitamento materno. Revista Gaúcha de Enfermagem, Porto Alegre, RS, v. 31, n. 2, p. 343-50, jun. 2010.

MACHADO, A. K. F.; ELERT, V. W.; PRETTO, A. D. B.; PASTORE, C. A. Intenção de amamentar e de introdução de alimentação complementar de puérperas. Ciência \& Saúde Coletiva, Rio de Janeiro, v. 19, n. 7, p. 1983-9, jul. 2014.

MARINHO, L. M. F.; CAPELLI, J. C. S.; ROCHA, C. M. M.; BOUSKELA, A.; CARMO, C. N. do; FREITAS, S. E. A. P.; ANASTÁCIO, A. S.; ALMEIDA, M. F. L. de; PONTES, J. S. Situação da alimentação complementar de crianças entre 6 e 24 meses assistidas na Rede de Atenção Básica de Saúde de Macaé, RJ, Brasil. Ciência \& Saúde Coletiva, Rio de Janeiro, v. 21, n. 3, p. 977-86, mar. 2016.

ORGANIZAÇÃO DAS NAÇÕES UNIDAS (ONU). Apenas 40\% das crianças são alimentadas exclusivamente com leite materno nos 6 primeiros meses de vida. 
2017. Disponível em: <https://nacoesunidas.org/apenas-40-das-criancas-saoalimentadas-exclusivamente-com-leite-materno-nos-6-primeiros-meses-de-vida/>. Acesso em: 23 ago. 2017.

ORGANIZAÇÃO MUNDIAL DE SAÚDE (OMS). Diretriz: ingestão de açúcares por adultos e crianças. Genebra, Suíça, 2015.

PALMEIRA, P.; CARNEIRO-SAMPAIO, M. Immunology of breast milk. Revista Associação Médica Brasileira, São Paulo, v. 62, n. 6, p. 584-93, jul. 2016.

PINO, J. L.V.; LÓPEZ, M. A. E.; MEDEL, A. P. I.; ORTEGA, A. S. Factores que inciden em laduración de lalactancia materna exclusiva en una comunidade rural de Chile. Revista Chilena de Nutrición, Santiago, Chile, v. 40, n. 1, p. 48-54, mar. 2013.

PRADO, C. V. C.; FABBRO, M. R. C.; FERREIRA, G. I. Desmame precoce na perspectiva de puérperas: uma abordagem dialógica. Texto Contexto Enfermagem, Florianópolis, v. 25, n. 2, p. 1-9, fev. 2016.

SÁ, N. N. B. de; GUBERT, M. B.; SANTOS, W. dos; SANTOS, M. P. Fatores ligados aos serviços de saúde determinam o aleitamento materno na primeira hora de vida no Distrito Federal, Brasil, 2011. Revista Brasileira de Epidemiologia, São Paulo, v. 19, n. 3, p. 509-24, jul./set. 2016.

SIMON, V. G. N.; SOUZA, J. M. P.; SOUZA, S. B. Breastfeeding, complementary feeding, overweight and obesity in pre-school children. Revista de Saúde Pública, São Paulo, v. 43, n. 1, p. 60-9, jul. 2009.

SOUZA, F. I. S. de; CAETANO, M. C.; ORTIZ, T. T.; SILVA, S. G. L. da; SARNI, R. O. $S$. Complementary feeding of infants in their first year of life: focus on the man pureed baby foods. Revista da Associação Médica Brasileira, São Paulo, v. 60, n. 3, p. 231-5, jan. 2014

TAMASIA, G. A.; VENÂNCIO, S. I.; SALDIVA, S. R. D. M. Situation of breastfeeding and complementary feeding in a médium-sized municipality in the Ribeira Valley, São Paulo. Revista de Nutrição, Campinas, SP, v. 28, n. 2, p. 143-53, mar./abr. 2015.

TOLONI, M. H. A.; LONGO-SILVA, G.; GOULART, R. M. M.; TADDEI, J. A. A. C. Introdução de alimentos industrializados e de alimentos de uso tradicional na dieta de crianças de creches públicas no município de São Paulo. Revista de Nutrição, Campinas, SP, v. 24, n. 1, p.61-70, jan./fev. 2011.

VICTORA, C. G.; BAHL, R.; BARROS, A. J. D.; FRANÇA, G. V. A.; HORTON, S.; KRASEVEC, J.; MURCH, S.; SANKAR, M. J.; WALKER, N.; ROLLINS, N. C. Breastfeeding in the 21st century: epidemiology, mechanisms, and lifelong effect. Lancet, England, v. 387, n. 10017, p. 475-90, jan. 2016. 